\title{
Paratireoidectomia na DRC
}

\author{
Parathyroidectomy in CKD
}

\section{Autores:}

Elisa de Albuquerque

Sampaio

Rosa Maria Affonso

Moysés
1 Indicações de Paratireoidectomia (PTx)

A PTx deve ser indicada em pacientes com doença renal crônica estágios III-V D e I-V T com hiperparatireoidismo secundário (HPS) ou terciário, respectivamente, não responsivo ao tratamento clínico, assim especificado:

1.1 Pacientes com HPS com nível sérico de PTH, persistentemente acima de $800 \mathrm{pg} / \mathrm{mL}$, associado a uma ou mais das seguintes condições:

1.1.1 Hipercalcemia e/ou hiperfosfatemia refratárias ao tratamento clínico (Evidência).

1.1.2 Hipercalcemia e/ou hiperfosfatemia durante pulsoterapia com calcitriol ou análogos da vitamina $\mathrm{D}$, a despeito do uso de quelante de $\mathrm{P}$ sem $\mathrm{Ca}$ e da redução da concentração de $\mathrm{Ca}$ do dialisato (Evidência).

1.1.3 Calcificações extraósseas (tecidos moles e/ou cardiovasculares) ou arteriolopatia urêmica calcificante (calcifilaxia) (Evidência).

1.1.4 Doença óssea avançada, progressiva e debilitante que não responde ao tratamento clínico (Evidência).

1.1.5 Presença de glândulas paratireoides volumosas ao ultrassom (volume $>1,0 \mathrm{~cm}^{3}$ ) (Opinião).

1.2 Pacientes com HP terciário, quando:

1.2.1 Associado à hipercalcemia maligna $($ Ca total $>14 \mathrm{mg} / \mathrm{dL}$ ou Ca iônico $>1,80 \mathrm{mmol} / \mathrm{L}$ ) (Evidência).
1.2.2 Associado a hipercalcemia e perda progressiva e inexplicada da função do enxerto (Evidência).

1.2.3 Hipercalcemia persistente após o primeiro ano de transplante renal.

\section{Avaliação pré-operatória}

2.1 Identificar as glândulas paratireoides através de ultrassonografia e cintilografia com sestamibi${ }^{99 \mathrm{~m} T c}$ (Opinião).

2.2 Descartar intoxicação alumínica nos pacientes com HPS, por meio do teste a desferroxamina (Evidência).

2.2.1 Nos casos de alta probabilidade dessa associação e na presença de um teste negativo ou duvidoso com desferroxamina, realizar biópsia óssea (Evidência).

\section{Tipos de PTx}

3.1 A PTx deve ser subtotal ou total com autoimplante de tecido paratireoideano (Evidência).

3.1.1 Nos casos de autoimplante de tecido paratireoideano, este pode ser realizado no antebraço ou na região pré-esternal (Opinião).

4 Tratamento da síndrome da fome óssea no pós-operatório imediato

4.1 Dosar potássio duas vezes ao dia, durante as primeiras 24 horas subsequentes à PTx. Dosar Ca sérico pelo menos duas vezes ao dia até a estabilização dos seus 
níveis e alta hospitalar. Em pacientes transplantados ou em tratamento conservador, monitorar também $\mathrm{P}$ e magnésio na mesma frequência (Opinião).

4.2 Iniciar infusão de gluconato de Ca IV imediatamente após o término da PTx. Utilizar 10 ampolas de gluconato de $\mathrm{Ca}$ a $10 \%$ diluídas em $250 \mathrm{~mL}$ de solução fisiológica a 0,9\%, infundido preferencialmente em veia calibrosa, na velocidade de $10 \mathrm{~mL} / \mathrm{h}$ através de bomba de infusão contínua. Posteriormente, a velocidade de infusão deve ser ajustada para manter o Ca sérico $\geq 7,5 \mathrm{mg} / \mathrm{dL}$ ou $\mathrm{Ca}$ iônico $\geq 1,0 \mathrm{mmol} / \mathrm{L}$ (Opinião).

4.2.1 Fazer uma dose suplementar de gluconato de $\mathrm{Ca}$ (uma ampola de gluconato de $\mathrm{Ca}$ a $10 \%$ IV, diluída em $50 \mathrm{~mL}$ de glicose a $5 \%$, em 10 minutos) sempre que o Ca sérico estiver $<7,5 \mathrm{mg} / \mathrm{dL}(<1,0 \mathrm{mmol} / \mathrm{L})$ ou o paciente apresentar sintomas de hipocalcemia (Opinião).

4.3 Iniciar carbonato de Ca na dose de $48 \mathrm{~g} / \mathrm{dia}$ ( 1 colher de sopa = 12 gramas), a cada 6 horas, por via oral, após a liberação da dieta, longe das refeições, ajustando de acordo com o Ca sérico (Opinião).

4.4 Iniciar calcitriol oral na dose de 2,5 $\mu \mathrm{g} / \mathrm{dia}$, fracionada em duas tomadas diárias, concomitante ao uso de carbonato de $\mathrm{Ca}$, ajustando de acordo com o Ca sérico (Opinião).

4.5 Após o segundo pós-operatório, as doses de carbonato de $\mathrm{Ca}$ e calcitriol devem ser ajustadas visando à suspensão da infusão de gluconato de $\mathrm{Ca}$, o mais precocemente possível (Opinião).

4.6 Em pacientes transplantados renais, as doses de gluconato de $\mathrm{Ca}$, carbonato de $\mathrm{Ca}$ e calcitriol devem ser reduzidas à metade do recomendado nos itens 4.2-4.4. (Opinião).

4.7 Após a PTx, usar dialisato com concentração de $\mathrm{Ca}$ de $3,5 \mathrm{mEq} / \mathrm{L}$ (Opinião).

\section{Cuidados no pós-operatório tardio}

5.1 Monitorar $\mathrm{Ca}$ e $\mathrm{P}$ séricos semanalmente, nas primeiras 4 semanas, após a alta hospitalar, e quinzenalmente até o término da fome óssea (Opinião).

\section{RACIONAL}

O HPS é uma complicação frequente em pacientes com DRC, contribuindo para as altas taxas de morbimortalidade dessa população. Ao longo do curso da DRC, o HPS requer monitoração e medidas de prevenção e tratamento enérgicas, as quais nem sempre são satisfatórias para seu adequado controle, levando à necessidade de tratamento cirúrgico através da PTx. ${ }^{1-3}$

Devido à falta de estudos randomizados e controlados avaliando tratamento clínico vs. cirúrgico do HPS avançado, torna-se difícil comparar seus benefícios em longo prazo. ${ }^{2}$ Ressalta-se que o surgimento de novas estratégias terapêuticas, como os calcimiméticos, podem vir a reduzir a necessidade de PTx. ${ }^{4}$ Entretanto, a PTx cirúrgica permanece a terapia definitiva para o HPS grave refratário ao manejo clínico. ${ }^{2}$

Os métodos de imagem para a localização das glândulas paratireoides antes da PTx nem sempre são suficientemente sensíveis, sendo a ultrassonografia e a cintilografia de paratireoides consideradas métodos complementares. ${ }^{5-7}$ Esses procedimentos facilitam a abordagem cirúrgica, apesar de a não visualização de qualquer glândula paratireoide não contraindicar a PTx.

São três tipos de PTx: a subtotal, a total e a total com autoimplante de tecido paratireoideano. ${ }^{8-14}$ Embora a escolha de um tipo ou outro dependa da experiência e da habilidade do cirurgião, atualmente tem-se optado pela PTx subtotal ou total com autoimplante em razão da alta taxa de hipoparatireoidismo resultante da PTx total. ${ }^{13}$ Não existe evidência que a PTx total com autoimplante seja superior ou inferior à PTx subtotal. A PTx total sem autoimplante não deve ser realizada em pacientes transplantados ou naqueles que estão na lista de espera para o transplante renal. ${ }^{2}$

Atualmente, alguns centros utilizam a dosagem do PTH intraoperatório como ferramenta para monitoração da efetividade da PTx. ${ }^{15-17}$

$\mathrm{O}$ autoimplante de tecido paratireoideano pode ser realizado tanto no antebraço como na região pré-esternal, dependendo da experiência do cirurgião. Com relação à PTx subtotal, geralmente o cirurgião escolhe como glândula remanescente aquela de menor tamanho e de melhor aspecto, deixando-a inteira ou efetuando ressecção parcial. Essa glândula remanescente é fixada com fio não reabsorvível para facilitar futuras intervenções em caso de recidiva. ${ }^{12}$ 
Após a PTx bem-sucedida, segue um período conhecido como "síndrome da fome óssea", que ocorre geralmente nos primeiros dias de pós-operatório, mas que, não raras vezes, surge tardiamente. As principais características dessa fase são hipocalcemia, hipofosfatemia e elevação da fosfatase alcalina total e óssea. Nessa fase, uma grande reposição de $\mathrm{Ca}$ e de calcitriol se faz necessária, por um período que varia desde os primeiros dias de pós-operatório até mesmo meses após a alta do paciente. ${ }^{17}$ Embora a reposição intravenosa de grandes quantidades de Ca seja objeto de controvérsia na literatura, a maioria dos pacientes, principalmente aqueles com HPS grave, desenvolve hipocalcemia sintomática, necessitando de tal medida. ${ }^{18,19}$

A administração de Ca e de calcitriol por via oral, tentando manter o Ca sérico na faixa normal, deve ser instituída o mais rapidamente possível, porque além de favorecer a redução dos episódios de hipocalcemia e suspensão mais rápida da infusão venosa de Ca, possibilitará menor tempo de hospitalização. ${ }^{20-22}$ Durante o período de "fome óssea", atenção especial deve ser dada às dosagens do potássio sérico, pois um significativo percentual desses pacientes desenvolve hipercalemia no pós-operatório imediato, inclusive necessitando de diálise emergencial. ${ }^{19,23,24}$

Embora a causa da hipercalemia pós-PTx venha sendo atribuída à maciça apoptose de osteoclastos, sua real gênese ainda é obscura na literatura. ${ }^{19}$ Além disso, alguns pacientes, principalmente aqueles com DRC pré-dialítica ou transplantados, desenvolvem hipomagnesemia, sendo essa complicação, muitas vezes, a causa da hipocalcemia sustentada no pós-operatório. ${ }^{25}$ A reposição de magnésio é feita com sulfato de magnésio intravenoso ou pindolato de magnésio por via oral, até que seus níveis voltem à normalidade. Quanto à reposição de P para correção da hipofosfatemia, esta deve ser evitada, exceção feita no caso de hipofosfatemia grave e sintomática, em que o nível sérico de $\mathrm{P}$ encontra-se abaixo de $1,0 \mathrm{mg} / \mathrm{dL}^{2}{ }^{26}$

Após o primeiro mês da PTx, é essencial a monitoração mensal de $\mathrm{Ca}$ e $\mathrm{P}$ séricos visando à modificação da posologia do $\mathrm{Ca}$ oral e do calcitriol. O Ca oral, inicialmente utilizado como suplemento, deve ter sua dose ajustada de acordo com as necessidades individuais. No decorrer do período pós-operatório tardio, a necessidade da mudança do Ca oral da forma de suplemento para a forma quelante, ou mesmo uma associação de ambas, deve ser sempre considerada. Às vezes, a introdução de quelantes não contendo Ca, como o sevelamer, em substituição ou como coadjuvante do $\mathrm{Ca}$, se faz necessária. Finalmente, a monitoração do PTH deve ser trimestral para identificação e intervenção precoces de possíveis elevações do hormônio e, também, para intervenção no caso de níveis muito reduzidos. Essas medidas são importantes na prevenção de recidivas e detecção precoce de persistência do HPS ou mesmo do hipoparatireoidismo e suas consequências.

\section{RefERÊNCIAS}

1. K/DOQI Clinical practice guidelines for bone metabolism and disease in chronic kidney disease. Guideline 14. Parathyroidectomy in patients with CKD. Am J Kidney Dis 2003; 23:S127-9. Disponível em: http://www.kidney. org/professionals/kdoqi/guidelines_bone/guide14.htm

2. Kidney Disease. Improving Global Outcomes (KDIGO) CKD-MBD Work Group. KDIGO clinical practice guideline for the diagnosis, evaluation, and treatment of chronic clinical disease-mineral and bone disorder (CKD-MBD). Chapter 4.2: Treatment of abnormal PTH levels in CKD-MBD. Kidney Int 2009; 76(113):S50-99.

3. Uhlig K, Berns JS, Kestenbaum B et al. KDOQI US commentary on the 2009 KDIGO clinical practice guideline for the diagnosis, evaluation, and treatment of CKD-mineral and bone disorder (CKD-MBD). AJKD 2010; 55:773-99.

4. Cunningham J, Danese M, Olson K, Klassen P, Chertow GM. Effects of the calcimimetic $\mathrm{HCl}$ on cardiovascular disease, fracture, and health-related quality of life in secondary hyperparathyroidism. Kidney Int 2005; 68:1793-800.

5. Tomic Brzac H, Pavlovic D, Halbauer M, Pasini J. Parathyroid sonography in secondary hyperparathyroidism: correlation with clinical findings. Nephrol Dial Transplant 1989; 4:45-50.

6. Takebayashi S, Matsui K, Onohara Y, Hidai H. Sonography for early diagnosis of enlarged parathyroid glands in patients with secondary hyperparathyroidism. Am J Roentgenol 1987; 148:911-4.

7. Olaizola I, Zingraff J, Heuguerot C et al. [(99m)Tc]sestamibi parathyroid scintigraphy in chronic haemodialysis patients: static and dynamic explorations. Nephrol Dial Transplant 2000; 15:1201-6.

8. Tominaga Y, Tanaka Y, Sato K, Nagasaka T, Takagi H. Histopathology, pathophysiology, and indications for surgical treatment of renal hyperparathyroidism. Semin Surg Oncol 1997; 13:78-86.

9. Jofré R, López Gómez JM, Menárguez J et al. Parathyroidectomy: whom and when? Kidney Int Suppl 2003; (85):S97-100.

10. Katoh N, Nakayama M, Shigematsu T et al. Presence of sonographically detectable parathyroid glands predict resistance to oral pulsed-dose calcitriol treatment of secondary hyperparathyroidism. Am J Kidney Dis 2000; 35:465-8.

11. Rostaing L, Moreau-Gaudry X, Baron E, Cisterne JM, Monroziès-Bernadet P, Durand D. Changes in blood pressure and renal function following subtotal parathyroidectomy in renal transplant patient presenting with persistent hypercalcemic hyperparathyroidism. Clin Nephrol 1997; 47:248-55. 
12. Accetta P, Accetta I, Cruz EAS et al. Paratireoidectomia subtotal no tratamento do hiperparatireoidismo secundário à doença renal crônica. J Bras Nefrol 2006; 28:65-71.

13. Proye C, Carnaille B, Sautier M. Hyperparathyroidism in patients with chronic renal failure: subtotal parathyroidectomy or total parathyroidectomy with autotransplantation? Experience with 121 cases. J Chir (Paris) 1990; 127:136-40.

14. Gagné ER, Ureña P, Leite-Silva S et al. Short- and longterm efficacy of total parathyroidectomy with immediate autografting compared with subtotal parathyroidectomy in hemodialysis patients. J Am Soc Nephrol 1992; 3:1008-17.

15. Gioviale MC, Gambino G, Maione C et al. Use of monitoring intraoperative parathyroid hormone during parathyroidectomy in patients on waiting list for renal transplantation. Transplant Proc 2007; 39:1775-8.

16. Ohe MN, Santos RO, Kunii IS et al. Usefulness of intraoperative PTH measurement in primary and secondary hyperparathyroidism: experience with 109 patients. Arq Bras Endocrinol Metabol 2006; 50:869-75.

17. Nilsen FS, Haug E, Heidemann M, Karlsen SJ. Does rapid intraoperative parathyroid hormone analysis predict cure in patients undergoing surgery for primary hyperparathyroidism? A prospective study. Scand J Surg 2006; 95:28-32.

18. Cozzolino M, Gallieni M, Corsi C, Bastagli A, Brancaccio D. Management of calcium refilling post-parathyroidectomy in end-stage renal disease. J Nephrol 2004; 17:3-8.
19. Kaye M, Rosenthall L, Hill RO, Tabah RJ. Long-term outcome following total parathyroidectomy in patients with end-stage renal disease. Clin Nephrol 1993; 39:192-7.

20. Cruz DN, Perazella MA. Biochemical aberrations in a dialysis patient following parathyroidectomy. Am J Kidney Dis 1997; 29:759-62.

21. Nagakawa M, Emoto A, Nasu N et al. Calcium supplement necessary to correct hypocalcemia after total parathyroidectomy for renal osteodystrophy. Int J Urol 2000; 7:35-40.

22. Clair F, Leenhardt L, Bourdeau A et al. Effect of calcitriol in the control of plasma calcium after parathyroidectomy. A placebo-controlled, double-blind study in chronic hemodialysis patients. Nephron 1987; 46:18-22.

23. Mazzaferro S, Chicca S, Pasquali $M$ et al. Changes in bone turnover after parathyroidectomy in dialysis patients: role of calcitriol administration. Nephrol Dial Transplant 2000; 15:877-82.

24. Shpitz B, Korzets Z, Dinbar A et al. Immediate postoperative management of parathyroidectomized hemodialysis patients. Dial Transplant 1986; 15:507.

25. Torralbo A, Portoles J, Perez Perez AJ, Barrientos A. Hypomagnesemic hypocalcemia in chronic renal failure. Am J Kidney Dis 1993; 21:167-71.

26. Lentz RD, Brown DM, Kjellstrand CM. Treatment of severe hypophosphatemia. Ann Intern Med 1978; 89:941-4. 